\title{
ESTUDO DOS NÍVEIS DE IGG ESPECÍFICA PARA O PEPTÍDEO K18 DE PORPHYROMONAS GINGIVALIS ATCC33277 NA PERIODONTITE CRÔNICA.
}

\author{
Isis Carolina de Oliveira Cordeiro ${ }^{1}$; Isaac Gomes Filho²; Ellen Karla Nobre dos \\ Santos Lima ${ }^{3}$; Yuri Andrade de Oliveira ${ }^{4}$; Márcia Tosta Xavier ${ }^{5}$ e Soraya Castro \\ Trindade ${ }^{6}$ \\ 1. Bolsista PIBIC/CNPq, Graduanda em Odontologia, Universidade Estadual de Feira de Santana, \\ e-mail: isiscarolinaoc@gmail.com \\ 2. Orientador, Departamento de Saúde, Universidade Estadual de Feira de Santana, \\ e-mail: isuzart@gmail.com \\ 3. Participante do projeto, Programa de Pós-Graduação em Imunologia, Universidade Federal da Bahia, \\ e-mail: ellenobre@hotmail.com \\ 4. Participante do projeto, Graduando em Odontologia, Universidade Estadual de Feira de Santana, \\ e-mail: yuriandrade.odont@gmail.com \\ 5. Participante do projeto, Curso de Odontologia, Escola Bahiana de Medicina e Saúde Pública, \\ e-mail: mtxavier@bahiana.edu.br \\ 6. Coorientadora, Departamento de Saúde, Universidade Estadual de Feira de Santana, \\ e-mail: soraya.castrotrindade@gmail.com
}

PALAVRAS-CHAVE: lys-gingipaína; peptídeo sintético; resposta imune.

\section{INTRODUÇÃO}

As doenças periodontais acometem a população brasileira expressivamente (OPPERMANN et al., 2015) e influenciam outras condições sistêmicas, como doenças cardiovasculares e cerebrovasculares isquêmicas; diabetes mellitus; doenças pulmonares; nascimento prematuro e baixo peso ao nascimento (GOMES-FILHO et al, 2007; 2010; CATON et al., 2018).

Uma atenção especial deve ser dispensada à periodontite, uma doença inflamatória que acomete os tecidos de suporte do dente, podendo levar a reabsorção do osso alveolar, destruição de fibras colágenas e, por fim, a perda dentária (CATON, 2018). Apresenta etiologia multifatorial, tendo como um dos principais agentes etiológicos a presença de um biofilme disbiótico na região subgengival (MAYER et al, 2013; HAJISHENGALIS e LAMONT, 2014).

Dentre os microrganismos presentes no biofilme, destaca-se Porphyromonas gingivalis, bactéria intensamente proteolítica e indutora de inflamação (MAYER et al, 2013). É um patógeno-chave na disbiose oral, sendo o microrganismo mais estudado em sua relação com a periodontite crônica (HAJISHENGALLIS, 2012; HAJISHENGALIS e LAMONT, 2014).

$P$. gingivalis possui uma ampla gama de fatores de virulência, dentre eles, as proteases (gingipaínas) que degradam moléculas sinalizantes e citocinas (PRESHAW \& TAYLOR, 2011), contribuindo para a sua capacidade de induzir doença periodontal (TEUGHELS et al, 2011). Atualmente, são descritas, para P. gingivalis ATCC 33277, as Arg-gingipaínas (RgpA e RgpB) e a Lys-gingipaína (Kgp), que teve um dos seus peptídeos, K18, obtidos por ferramenta de imunoinformática, investigado no presente estudo.

Diante da importância da compreensão da interação microrganismo-hospedeiro na patogênese da periodontite, o presente trabalho pretende avaliar o reconhecimento do peptídeo K18 de lys-gingipaína de $P$. gingivalis ATCC33e77 por IgG de indivíduos com e sem periodontite. 


\section{MÉTODO}

A presente investigação está vinculada ao projeto "Análise in silico e in vitro de epítopos candidatos de Porphyromonas gingivalis ATCC 33277”. O peptídeo K18 foi obtido a partir de análise in silico realizada no referido projeto, que objetivou identificar, por bioinformática, peptídeos com potencial imunogênico em proteínas de $P$. gingivalis e, após a síntese química, testar in vitro tais peptídeos quanto a sua imunorreatividade e imunogenicidade.

A síntese do peptídeo K18 foi feita pela empresa Aminotech Pesquisa e Desenvolvimento Ltda (Diadema - SP). A solubilização do peptídeo liofilizado foi realizada em tampão de trabalho carbonato-bicarbonato $\mathrm{e}$ as alíquotas foram armazenadas a $-20^{\circ} \mathrm{C}$.

Para as análises de imunogenicidade, foi empregado um pool de soros de indivíduos com periodontite (controle positivo) e um pool de soros de indivíduos sem periodontite (controle negativo). Foram selecionados 21 indivíduos com periodontite e 21 indivíduos sem a doença, todos com idade igual ou superior a 18 anos, que buscaram atendimento no ambulatório da disciplina Estudos Integrados IX do Curso de Odontologia da Universidade Estadual de Feira de Santana (Feira de Santana - BA). Os critérios avaliados pela anamnese para a não inclusão dos voluntários foram número de dentes inferior a 10; história de doenças sistêmicas; gestação atual; tratamento periodontal anterior ( 1 ano); tabagismo atual ou anterior; etilismo atual ou anterior; uso de antibióticos e anti-inflamatórios, respectivamente, nos seis e dois meses anteriores à coleta. Os voluntários assinaram o termo de consentimento livre e esclarecido em duas vias. O diagnóstico periodontal foi realizado com base nos critérios preconizados por GOMES-FILHO et al. (2007).

O soro dos participantes foi obtido após coleta de sangue por punção venosa na fossa antecubital em tubos para coleta a vácuo (BD, SP, Brasil) com ativador de coagulação. Os soros foram testados quanto à sua imunorreatividade ao extrato sonicado de Porphyromonas gingivalis (TRINDADE et al., 2008).

Para a composição dos pools, os indivíduos foram selecionados de acordo com o diagnóstico periodontal e com a reatividade do soro. Assim, o pool controle positivo foi composto pelo soro de seis indivíduos com diagnóstico de periodontite e com os maiores valores de densidade ótica obtidos nos ensaios com o extrato sonicado de Porphyromonas gingivalis. O pool controle negativo foi composto pelo soro de cinco indivíduos sem a referida doença e com os menores valores de densidade ótica obtidos nos ensaios com o extrato sonicado de Porphyromonas gingivalis.

A imunorreatividade dos pools ao peptídeo K18 foi testada por ensaio imunoenzimático, padronizando-se as concentrações de antígeno, anticorpo de detecção conjugado com peroxidade e a diluição dos pools de soro (ELISA checkboard). A concentração de IgG foi mensurada por leitura da densidade ótica (D.O.) em leitor de ELISA padrão. con

Os valores de densidade óptica obtidos com os pools controle positivo foram divididos pelos valores do pool controle negativo (Média Grupo PC/Média Grupo SP), para a obtenção de um coeficiente que determina a melhor diferenciação de resposta entre os grupos.

\section{RESULTADOS E/OU DISCUSSÃO}

Os soros testados apresentaram imunorreatividade ao peptídeo K18, com o reconhecimento deste peptídeo por IgG específica. Das condições de estudo testadas no imunoensaio enzimático (ELISA), a utilização do K18 com os pools de soro diluídos a 1:100 apresentou a melhor condição de diferenciação entre o pool de soros de indivíduos com periodontite (controle positivo) e o pool de soros de indivíduos sem a 
doença (controle negativo), com um coeficiente (DO controle positivo/ Do controle negativo) de 1.8 (Gráfico 01). Este dado indica que o reconhecimento de K18 por IgG no controle positivo foi $80 \%$ maior (em densidade ótica) que no controle negativo.

Os valores de coeficiente observados nos experimentos com o extrato sonicado de Porphyromonas gingivalis foram iguais (soro 1:250) ou inferiores (soro 1:100) aos encontrados com o K18, possivelmente pela grande variedade de antígenos existente no extrato, favorecendo a ocorrência de reatividade cruzada, principalmente no pool controle negativo (Franca et al., 2007; Trindade et al., 2008). A utilização de proteínas/peptídeos isolados, que apresentem um bom potencial de imunorreatividade, parece ser mais adequado para o emprego em imunoensaios que visem o conhecimento da resposta imune humoral (Trindade et al., 2012).

Diante do exposto, o emprego do peptídeo K18 se mostrou promissor para análises de imunorreatividade futuras. Para isto, são necessários ensaios com a utilização de amostras individuais de soro, utilizando grupos de indivíduos com e sem periodontite com tamanho de amostra suficiente para a comparação estatística entre eles. Sabendo-se que os fatores de virulência podem induzir a produção de citocinas pró e antiinflamatórias, considera-se que estudar tais moléculas seja importante na compreensão dos mecanismos patogênicos da periodontite. Logo, a analise in silico possibilita que peptídeos promissores na aplicação biotecnológica sejam identificados, assim como sua interação com o hospedeiro (BITTNER-EDDY et al., 2013). A utilização da analise in silico junto com ferramentas de imunodiagnósticos, como por exemplo a avaliação do reconhecimento de peptídeos sintéticos obtidos a partir da análise in silico por IgG humana específica, pode auxiliar no estudo dos fatores de virulência e na busca pelo entendimento da patogenicidade de $P$. gingivalis.

A realização de estudos que fortaleçam o entendimento da interação patógenohospedeiro é de grande relevância na periodontite, uma doença inflamatória que pode levar a diversos agravos na saúde do indivíduo (CATON et al., 2018), cuja relação com estes agravos pode ter a sua plausibilidade biológica pautada na referida interação patógeno-hospedeiro.

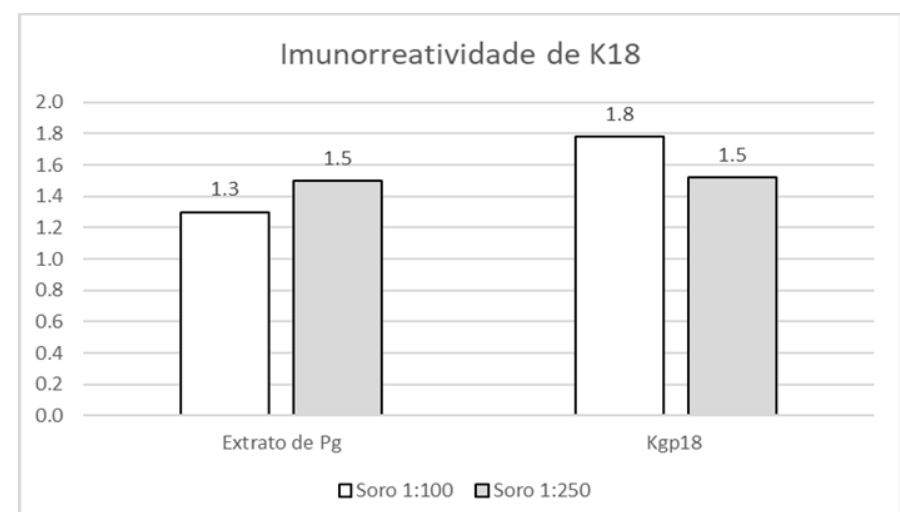

Gráfico 01: Coeficiente entre as médias da D.O. obtidas para cada grupo (Média Grupo PC/Média Grupo SP) após análise da reatividade à $\mathrm{I} / \mathrm{gG}$ do peptídeo $\mathrm{K} 18$. Este foi reconhecido por IgG específica presente no soro dos participantes.

\section{CONCLUSÃO}

O peptídeo K18 possui potencial imunogênico e foi selecionado para sua utilização em estudos in vitro subsequentes. 


\section{REFERÊNCIAS}

BITTNER-EDDY, P.D.; FISCHER, L.A.; COSTALONGA, M. Identification of gingipain-specific $\mathrm{I}^{\mathrm{b}} \mathrm{b}^{\mathrm{b}}$-restricted $\mathrm{CD} 4^{+} \mathrm{T}$ cells following mucosal colonization with Porphyromonas gingivalis in C57BL/6 mice. Molecular oral microbiology, v. 28, p. 452-66, 2013.

CATON, J.G.; ARMITAGE, G.; BERGLUNDH, T.; CHAPPLE, I.L.C.; JEPSEN, S.; KORNMAN, K.S. et al. A new classification scheme for periodontal and peri-implant diseases and conditions - Introduction and key changes from the 1999 classification. J Clin Periodontol 2018;45(Suppl 20): S1-S8.

Franca M, Moura-Costa L, Meyer RJ, Trindade SC, Tunes UR, Freire SM. Humoral immune response to antigens of Porphyromonas gingivalis ATCC 33277 in chronic periodontitis. J Appl Oral Sci 2007;15(3):213-9.

GOMES-FILHO, I.S.; CRUZ, S.S.; REZENDE, E.J.; DOS SANTOS, C.A.; SOLEDADE, K.R.; MAGALHÃES, M.A.; DE AZEVEDO, A.C.; TRINDADE, S.C.; VIANNA, M.I.; PASSOS, J.S.; CERQUEIRA, E.M. Exposure measurement in the association between periodontal disease and prematurity / low birth weight. J Clin Periodontol., v. 34, n. 11, p. 957-63, 2007.

GOMES-FILHO, I.S.; CRUZ, S.S.; COSTA, M.C.N.; PASSOS, J.S.; CERQUEIRA, E.M.M.; SAMPAIO, F.P.; PEREIRA, E.C.; MIRANDA, L.F. Periodontal therapy and low birth weight: preliminary results from an alternative methodologic strategy. Journal of Periodontology, v. 81, n. 12, p. 1725-33, 2010.

HAJISHENGALLIS, G.; LAMBRIS, J.D. Complement and dysbiosis in periodontal disease. J. Immunobiology, v. 217, p. 1111-1116, 2012.

HAJISHENGALLIS, G.; LAMONT, R. Breaking bad: Manipulation of the host response by Porphyromonas gingivalis. Trends in Immunology, v.44, p. 328-338, 2014.

MAYER, M.P.A.; SUGUIMOTO, E.S.A.; TEIXEIRA, S.R.L. Microbiologia da doença periodontal. In: SPOLIDORIO, D.M.P.; DUQUE, C. Microbiologia e imunologia geral e odontológica. v.1. São Paulo: Artes Médicas, 2013. p. 91-99.

OPPERMANN, R.V; HAAS, A. N; RÖSING, C. K; SUSIN, C. Epidemiology of periodontal diseases in adults from Latin America. J. Periodontology 2000. 2015; 67:13-33.

PRESHAW, P.M.; TAYLOR, J.J. Patogênese periodontal. In: NEWMAN, M.G.; TAKEI, H.; KLOKKEVOLD, P.R.; CARRANZA, F.A. Carranza, periodontia clínica. 11ed. Rio de Janeiro: Elsevier, 2011. p. 210-35.

TEUGHELS, W.; QUIRYNEN, M.; JAKUBOVICS, N. Microbiologia periodontal. In: NEWMAN, M.G.; TAKEI, H; KLOKKEVOLD, P.R.; CARRANZA, F.A. Carranza, periodontia clínica. 11ed. Rio de Janeiro: Elsevier, 2011. p. 251-94.

Trindade SC, Gomes-Filho IS, Meyer RJ, Vale VC, Pugliese L, Freire SM. Serum antibody levels against Porphyromonas gingivalis extract and its chromatographic fraction in chronic and aggressive periodontitis. Journal of the international academy of Periodontology 2008;10(2):50-8.

Trindade SC, Olczak T, Gomes-Filho IS, Moura-Costa LF, Cerqueira EMM, GaldinoNeto M, Alves H, Carvalho-Filho PC, Xavier MT, Meyer R. Induction of interleukin (IL)-1b, IL-10, IL-8 and immunoglobulin G by Porphyromonas gingivalis HmuY in humans. J Periodont Res 2012;47:27-32. 